\title{
CODIMENSION OF SOME SUBSPACES IN A FRÉCHET ALGEBRA
}

\author{
JENS PETER REUS CHRISTENSEN
}

ABSTRACT. In a complete separable metrizable topological algebra, if the linear span of the set of all products of two elements has at most countable algebraic codimension, then it has finite codimension.

In the present note we give a partial solution of a question posed to us by P. C. Curtis. Furthermore we give some closely related remarks.

Let $\mathcal{Q}$ be an algebra over the real or complex number field. By $\mathbb{Q}^{2}$ we understand the linear span of all products of two elements. In the commutative case this coincides with the linear span of all squares. The original question of P. C. Curtis was about $\mathbb{Q}^{2}$; our theorem will contain both $\mathbb{Q}^{2}$ and the linear span of squares as very special cases. We obtain completely satisfying results for separable complete metrizable topological algebras (local convexity is completely unnecessary for the proofs), but, unfortunately, every attempt to establish our results for nonseparable algebras has failed so far. Before we start on the theorem, let us give the motivation for the question of P.C. Curtis, who asked whether the square of a maximal modular ideal in a Banach algebra is closed if it has finite codimension in the ideal (the question seems to have been raised independently by other people; see [4]).

If $\mathfrak{Q}$ is an algebra over the scalar field (real or complex numbers) and $\varphi$ is a multiplicative linear functional, then a point derivation $D$ at $\varphi$ is a linear functional satisfying the equation

$$
D(a b)=\varphi(a) D(b)+D(a) \varphi(b)
$$

for all $a, b \in \mathbb{Q}$. If $\mathbb{Q}$ has a unit then a linear functional $D$ on $\mathbb{Q}$ is a point derivation at $\varphi$ if and only if $D$ annihilates both the unit and the square of the kernel of $\varphi$. Consequently, for algebras with unit, the space of point derivations at some multiplicative linear functional $\varphi$ is finite dimensional if and only if the square of the kernel of $\varphi$ has finite codimension in the kernel. This equivalence does not hold for algebras without unit. However the conclusion drawn from the equivalence mentioned above and our affirmative answer to the question of P. C. Curtis (in the separable case), that finite dimensionality of the space of point derivations implies continuity of the point derivations, also holds for algebras without unit as we shall see.

THEOREM. Let $f_{n}$ be a sequence of polynomials in finitely many noncommuting

Received by the editors September 29, 1975.

AMS (MOS) subject classifications (1970). Primary 46H05, 46H15.

c) American Mathematical Society 1976 
variables over the scalar field. Let $S$ be the linear span of all values of the $f_{n}$ 's when elements of a complete separable metrizable topological algebra $\mathbb{Q}$ are inserted instead of the variables. Assume that the algebraic codimension of $S$ in $\mathbb{Q}$ is at most countable. Then $S$ is closed and its codimension in $\mathbb{Q}$ is finite. Furthermore, there are finitely many of the $f_{n}$ 's whose values span $S$, and for every set of finitely many $f_{n}$ 's whose values span $S$ there is an upper bound for the number of terms needed to write an element of $S$ as a linear combination of values of those finitely many polynomials.

Proof. We note that a complete metrizable topological algebra has simultaneous continuity of the multiplication if only separate continuity is assumed (the weakest reasonable definition of a topological algebra). This follows from a standard Baire category argument.

We choose a complemented subspace of $S$ of at most countable dimension. This subspace is $\sigma$-compact (as an at most countable union of finite dimensional subspaces). In particular this subspace is analytic (continuous image of a Polish space; for an introduction to the theory of analytic spaces and sets see the first three chapters of [1]). The space $S$ itself is a countable union of analytic sets, hence also analytic (this uses only the fact that $\mathbb{Q}$ is a Polish space (topologically complete separable metrizable space) and that multiplication is continuous). An application of Theorem 5.5 in [1] now shows that $S$ is closed and of finite codimension.

Let $A_{n} \subseteq S$ be the set of elements which are expressible as a linear combination of at most $n$ values of the polynomials $f_{v}, 1 \leqslant v \leqslant n$. The set $A_{n}$ is analytic, hence has the Baire property. (See [1, Chapter 2]. A subset of a Hausdorff topological space has the Baire property by definition if it differs from an open set by a set of first category. Since those sets form a $\sigma$-field we of ten use the term BP-measurable instead of "having the Baire property".) Under the assumptions of the theorem, $S$ is closed and therefore there exists $n$ such that $A_{n}$ is of the second category in $S$. Hence Pettis' lemma (see [1, Theorem 5.1]) shows that $A_{n}-A_{n}$ is a neighbourhood. Since this set is also a cone, it equals $S$. Hence there are finitely many of the $f_{n}$ 's whose values span $S$. Whenever we have finitely many of the $f_{n}$ 's whose values span $S$, an argument almost identical to the above argument shows that there is an upper bound for the number of terms needed to express an element of $S$ as a linear combination of those finitely many polynomials.

The reader should note that every important element in the above proof was well known before [1] appeared. The proof only needs some comparatively elementary facts from the theory of analytic (even metrizable) spaces. It is important, of course, that analytic sets are stable under countable unions and that a mapping between analytic spaces whose graph is analytic (in the proof of the graph of a projection operator) is in fact Borel measurable. Also important is the fact that an analytic set has the Baire property and the Pettis lemma. All necessary information on analytic sets and spaces can be found in [2]. The appendix of [3] seems to contain everything needed for the proof (local convexity as assumed in the appendix is superfluous). This finishes the proof of the theorem.

It is apparently not known whether the assumption of continuity of the functional $\varphi$ in the following corollary is automatically fulfilled. Only if $\mathscr{Q}$ is a 
Banach algebra is this continuity assumption therefore superfluous.

COROllary. Let $\varphi$ be a continuous multiplicative linear functional on the separable metrizable complete topological algebra $\mathbb{Q}$ over the scalar field. If the linear space of point derivations of $\mathbb{Q}$ at $\varphi$ is of at most countable dimension, then the dimension of this space is finite and it consists of continuous functionals.

Proof of Corollary. If $\mathscr{Q}$ has a unit, the corollary follows immediately from the theorem modulo the remarks preceding the theorem. If $Q$ has no unit, we just add a unit and, since both $\varphi$ and point derivations at $\varphi$ extend uniquely, the proof is finished.

The only nonseparable result we have been able to obtain so far is that if $\mathscr{U}$ is a Von Neumann algebra acting on a separable Hilbert space, then our theorem is valid and $S$ is even ultraweakly closed. The proof is considerably deeper than the above proof and we omit the details. It uses Theorem 5.8 in [1] and a result of Takesaki that ultraweak continuity on commutative selfadjoint subalgebras implies global ultraweak continuity (for linear functionals).

AcKnowledgements. The author is thankful to P. C. Curtis and K. B. Laursen for stimulating and encouraging discussions.

\section{REFERENCES}

1. J.P.R. Christensen, Topology and Borel structure, North-Holland Math. Studies, vol. 10; Notas de Matemática, no. 51, North-Holland, Amsterdam; American Elsevier, New York, 1974. MR 50 \# 1221.

2. C. Kuratowski, Topologie. Vol. 1, 2nd ed., Monografie Mat., Tom 20, PWN, Warsaw, 1948. MR 10, 389.

3. F. Trèves, Topological vector spaces, distributions and kernels, Academic Press, New York and London, 1967. MR 37 \#26.

4. Problems list from the conference on derivations and homomorphisms at U.C.L.A., July 1974.

KobenhaVns Universitets Matematiske Institut, Universitetsparken 5, 2100 CopenhaGEN $\varnothing$, DENMARK 